\title{
Von der Notwendigkeit der Verknüpfung von National- und Fremdsprachen im Prozess der Alphabetisierung und der informellen Bildung in Burkina Faso
}

\author{
Jean-Claude Bationo \\ Ecole normale supérieure, Université de Koudougou, BP. 376 Koudougou, Burkina Faso \\ E-mail: jclaude_bationo@yahoo.fr
}

\begin{abstract}
According to the 2006 national census 71,06\% of Burkina Faso's estimated 14 million inhabitants are functionally illiterate. This staggering number is referred to in explaining the slow social, economical and political development of the country; it is also linked to the high incidence of poverty in Burkina Faso. The government is aware of these connections between literacy and social circumstances and thus is determined to combat poverty through a literacy campaign. This article describes some of these attempts and critically reflects on the importance of the mastery of literacy in national languages, linking this also to the teaching and learning of foreign languages.
\end{abstract}

Keywords: literacy, informal education, language policy, national languages, foreign languages, employment

Schlüsselbegriffe: .Alphabetisierung, informelle Bildung, Sprachenpolitik, Nationalsprachen und Fremdsprachen, Stellensuche

\section{Einleitung}

In Burkina Faso ist das Analphabetentum ein Hindernis für die Entwicklung des Landes. Nach der Volkszählung im Jahre 2006 zählte Burkina Faso 71,06\% Analphabeten. Wie Spezialisten der Entwicklungsbranche voraussagen, kann sich kein Land entwickeln, wenn dieses eine Alphabetisierungsrate von 40\% nicht übersteigt. Das Analphabetentum ist eines der Probleme, mit denen sich Entwicklungsinitiativen auseinandersetzen müssen. Die Ergebnisse einer von den Vereinten Nationen im Jahre 2007 durchgeführten Erhebung zum Entwicklungsstand klassifizieren Burkina Faso nach dem Human Development Index unter 177 Ländern auf dem 176. Rang. Somit ist Burkina Faso das zweitärmste Land der Welt (Kinda 2009:15).

Diese Einschätzung in der weltweiten Rangordnung der Länder stört die burkinische Regierung, weshalb sie eine zunehmende Alphabetisierung der Bevölkerung als Priorität ansieht. Durch die Entwicklung vieler Pläne, wie zum Beispiel dem zehnjährigen Plan zur Entwicklung der Grunderziehung (Le Plan Décennal de Développement de l'Education de Base (PDDEB)), dem Forum zur Alphabetisierung, der Gründung des Fonds zur Alphabetisierung und zur informellen Schule zeigt die Regierung ihr Interesse an der 
Bekämpfung des Analphabetentums bzw. der Armut. Solch ein Engagement der burkinischen Regierung ist im Rahmen der weltweiten Bekämpfung des Analphabetentums (vgl. Lepeytre und Parra-Ponce 2008: 46 ff., Geoffroy und Grasset-Morel 2003:17ff., Besse 2003:82ff.), der Aktion von UN-Organisationen wie der UNICEF und der UNESCO zur elementaren „Bildung für alle bis 2015“ und der Förderung einheimischer Sprachen in Ländern der westafrikanischen Region zu verstehen (vgl. UNESCO 2007:65ff.).

Der vorliegende Beitrag zielt darauf ab, einerseits eine Bilanz der Nationalsprachenpolitik in Burkina Faso zu ziehen und andererseits die aktuelle Politik der Professionalisierung der informellen Bildung zu reflektieren, nämlich der Gründung spezifischer Berufe im informellen Bildungssektor. Ferner wird untersucht, inwiefern die Beherrschung der einheimischen Sprachen dazu beitragen kann, das Analphabetentum zu bekämpfen und Fremdsprachen besser zu lernen. Deshalb sollen zunächst einige Grundkonzepte wie Alphabetisierung und informelle Bildung erläutert werden. Dann erfolgt einerseits eine Analyse der Forumsbilanz zur „Erziehung für alle bis 2015“ besonders in Burkina Faso und andererseits eine kritische Darstellung der Bemühungen des burkinischen Staates und NichtRegierungsorganisationen im Bereich der Alphabetisierung. Diese Bilanz führt zur Lehrkraftsproblematik für einheimische Sprachen, die mit Überlegungen über eine mögliche Berufsorientierung im informellen Bildungssektor schließt.

\section{Konzepterläuterung}

Zum besseren Verständnis der gegebenen Problematik ist es notwendig, einige Konzepte zu erläutern. Das Konzept der Alphabetisierung hat sich sehr schnell entwickelt, so dass man heute zwischen einer traditionellen und funktionellen Alphabetisierung unterscheidet. Etymologisch besteht das Konzept Alphabetisierung in der Fähigkeit, das Alphabet einer bestimmten Sprache zu lesen und zu schreiben. Dem französischen Universalwörterbuch (1996:45) nach bedeutet der Begriff Alphabetisierung, Analphabeten das Schreiben und das Lesen zu lehren. Das Gesetz 013-2007/AN vom 30. Juli 2007 bezüglich des Erziehungsgesetzes in Burkina Faso definiert die Alphabetisierung in seinem Artikel 2 wie folgt:

L'ensemble des activités éducatives et de formation destinées à des jeunes et des adultes en vue d'assurer l'acquisition des compétences de base dans une langue donnée et qui vise l'autonomie de l'apprenant. Elle est une composante de l'éducation non formelle.

Diese Definition der Alphabetisierung geht über die einfache Fähigkeit des Lesens und des Schreibens hinaus. Nach der burkinischen Verfassung bedeutet Alphabetisierung „Erziehung und Ausbildung“. Dies zeigt die Entwicklung des Konzeptes Alphabetisierung, so dass es relevant ist, die Unterscheidung zwischen den traditionellen und den funktionalen Formen des Begriffes nachvollziehen zu können.

Die Alphabetisierung ist traditionell, wenn sie darauf abzielt, die Betroffenen von der Ignoranz gegenüber der Vermittlung von Lese-, Schreib- und Rechenfähigkeiten zu befreien, ohne eine besondere Orientierung zu bieten, ohne wissenschaftliche Vermittlungsmethoden anzuwenden, ohne Zukunftsaussichten zu liefern, ohne die Interessen der Alphabetisierten und die Investitionsmöglichkeiten $\mathrm{zu}$ betrachten, die nach einer Alphabetisierung zur 
Verbesserung der Lebensqualität beitragen können. Bei einem solchen Verständnis von Alphabetisierung fehlt eine Planung von staatlicher oder institutioneller Seite. Tatsächlich erlaubt sie keine Entwicklung von Kompetenzen, die dem Alphabetisierten zugute kommt und die er nach der Ausbildung wieder investieren bzw. das Gelernte wieder benutzen kann. Solch eine Alphabetisierung ist quantitativ und zielt darauf ab, mehr Menschen zu erreichen, anstatt diese darauf vorzubereiten, sich der Erweiterung ihres eigenen Bildungsniveaus bewusst zu werden. Sie hat auch zum Ziel, die Analphabetenrate zu reduzieren, anstatt jedem Alphabetisierten die Weltprobleme zugleich erklären und dadurch einen verantwortlichen Menschen ausbilden zu wollen.

Danach definiert die „Interregierungsagentur der Frankophonie“ die traditionelle Alphabetisierung wie folgt:

„un type d'alphabétisation dont l'objectif est l'enseignement de la lecture, du calcul et de l'écriture dans une langue donnée, afin de permettre aux néoanalphabètes d'accéder à la communication écrite ou imprimée dans une langue" (vgl. Kinda 2009:35).

Darüber hinaus wird hier mit Nachdruck betont, dass diese Alphabetisierungsform zwar die Analphabetenquote vorübergehend reduziert, ohne jedoch den langfristigen Entwicklungsstand des Landes stark zu beeinflussen. Ihr fehlt es an erkennbaren Folgen und einer Vertiefung und lässt daher die Begünstigten wieder ins Analphabetentum verfallen.

Dem steht die Rede von einer funktionalen Alphabetisierung gegenüber, die im Gegensatz zur traditionellen Alphabetisierung darin besteht, die Alphabetisierungsaktivitäten in den Entwicklungsprozess zu integrieren. Die funktionale Alphabetisierung unterscheidet sich von der traditionellen Alphabetisierung dadurch, dass sie wissenschaftlich fundiert ist. Sie ist objektiv geplant und richtet sich jeweils nach dem Lernniveau mit bestimmtem Inhalt, bestimmten Lehr- und Lernmethoden und mit klar definierten Zielsetzungen. Sie betont den Erwerb theoretischer und praktischer Kenntnisse und die Entwicklung von Fähigkeiten zur Durchführung bestimmter Arbeiten im Alltagsleben. Sie erlaubt, die Herausforderungen des sozialen Milieus der Alphabetisierten in Bezug auf ihre Interessen und die Erziehungszielsetzungen des Landes anzunehmen. Es reicht heute nicht mehr aus, lesen, schreiben und rechnen zu können, sondern man muss ein aktiver mündiger Bürger werden. Solch eine Alphabetisierung ist eine Fortschrittsalphabetisierung bzw. eine Entwicklungsalphabetisierung. In diesem Sinne kommt die Alphabetisierung dem Lernenden zugute, nämlich durch den Erwerb von Grundkompetenzen, wie z.B. die Welt lesen zu können, sie besser $\mathrm{zu}$ verstehen, um folgerichtiges und verantwortliches Verhalten $\mathrm{zu}$ erlernen.

In dem vorliegenden Beitrag geht es um die funktionale Alphabetisierung, obwohl es schwierig ist, diese von der traditionellen Alphabetisierung zu trennen. Die Alphabetisierung, sei sie traditionell oder funktionell, ist nach den Vereinten Nationen (UNESCO 2007:65) nicht nur ein Grundrecht des Menschen, sondern auch ein unentbehrliches Hilfsmittel bei der Entwicklung einer Gesellschaft.

Was die informelle Bildung anbelangt, wird sie vom Gesetz 013-2007/AN des 30. Juli 2007 als Bestandteil des Erziehungsgesetzes in Burkina Faso im Artikel 2 als alle strukturierten und 
organisierten Erziehungs- und Ausbildungsaktivitäten in einem nicht schulischen Rahmen definiert. Die informelle Bildung wendet sich an Jugendliche und die Erwachsene, die über 15 Jahre alt und nicht eingeschult worden sind. Sie ist ein Aspekt des Teilsystems der Grunderziehung und bezweckt die Ausrottung des Analphabetismus. Sie umfasst die Alphabetisierung und weitere Ausbildungsaktivitäten zur Verbesserung der Lebensbedingungen und zur Teilhabe der Ausgebildeten am Entwicklungsprozess. Die Hauptausbildungsstätten sind die Zentren der informellen Grunderziehung und die permanenten Alphabetisierungs- und Ausbildungszentren.

Da die einheimischen Sprachen eine sehr wichtige Rolle bei der Alphabetisierung spielen, werden im Nachstehenden einerseits die Beziehung zwischen einheimischen Sprachen und Alphabetisierung skizziert und andererseits die Erweiterung des Horizonts der Alphabetisierten durch das Lesen und das Schreiben von nationalsprachigen Zeitungen, Werken und Lehrwerken dargestellt.

\section{Nationalsprachen und Alphabetisierung}

Seit der Erlangung der Unabhängigkeit im Jahre 1960 hatte der Kampf gegen das Analphabetentum in Burkina Faso immer Priorität. 1961 wurden Zentren zur Landerziehung gegründet, die darauf abzielten, die jungen Landleute im Französischen zu alphabetisieren. Nach einer negativen Evaluierung dieses Projektes wurden diese Zentren 1974 abgeschafft.

1965 wurde die funktionale Alphabetisierung in einheimischen Sprachen mit dem Projekt „UNESCO-HAUTE-VOLTA“ ins Leben gerufen. Mit Hilfe von Kirchen, Vereinen und Nicht-Regierungsorganisationen hat sich die funktionale Alphabetisierung in den Nationalsprachen sehr schnell entwickelt. Diese Entwicklung erklärt sich dadurch, dass die Alphabetisierten Interesse daran haben, ihr Bildungsniveau anzuheben.

Landwirtschaft ist die Hauptaktivität der burkinischen Bevölkerung, wo neben Ackerbau auch Gemüseanbau, Fischfang und Viehzucht gehört. Die Landwirte, die von ihren täglichen Tätigkeiten leben wollen, sind meist Analphabeten und können daher über ihre Projekte weder in der eigenen noch in der Fremdsprache schreiben. Deshalb werden in allen Regionen Burkinas Alphabetisierungszentren gegründet, um Jugendliche und Erwachsene in den Nationalsprachen auszubilden und zu alphabetisieren. Um diese Ziele zu erreichen, werden nationalsprachige Lehrwerke so konzipiert, dass die Bedürfnisse und die täglichen Aktivitäten der Bevölkerung Beachtung finden. Diese bilden die Hauptthemen in den Lehrwerken.

Neben dem Lesen, Schreiben, Rechnen und Erzählen lernen die Alphabetisierten auch neue Anbautechniken in der einheimischen Sprache kennen. Sie werden ebenfalls für Grundfragen über die Entwicklung des Dorfes, der Region und des Landes sensibilisiert, indem sie Informationen über die Bevölkerungsprobleme bekommen, wie zum Beispiel Gesundheit, Umweltzerstörung und -schutz, Ernährung, Sexualität und Bevölkerungswachstum, um nur einige zu nennen. Diese Themen werden in den nationalsprachigen Zeitungen weiter verbreitet. Die Zeitungen in lokalen Sprachen verbessern tatsächlich die Ausbildung und die Sensibilisierung. Darüber hinaus lesen die Alphabetisierten in diesen Zeitungen allgemeine, soziale, politische, wirtschaftliche, religiöse und kulturelle Informationen. Häufig werden detaillierte Informationen über die Bodenerosion, das Herstellen von Kompost, die Benutzung von Dünger, die Ernteführung usw. geliefert. Dadurch werden nicht nur die Kenntnisse der 
Lerner über ihre täglichen Aktivitäten verbessert und erweitert, sondern sie entdecken auch weitere Welten. Die besten Alphabetisierten verfassen Zeitungsartikel in einheimischen Sprachen. Einige berichten in diesen Zeitungen von ihren Erfahrungen. Dieses Kommunikationsmittel erweist sich für sie außerdem als ein Weg, für ihre Produkte zu werben.

Während sich einige Alphabetisierten für das Verfassen von Zeitungsartikeln interessieren, beteiligen sich andere an der Produktion fiktiver Texte in Nationalsprachen. So werden Sagen, Märchen, Sprichwörter, Erzählungen und Theaterstücke von den Alphabetisierten niedergeschrieben. Das Lesen dieser Texte in einheimischen Sprachen erweitert den Horizont der Alphabetisierten. Ferner verhelfen die Informationen bzw. die Inhalte der nationalsprachigen Werke den Lernern zur Entfaltung ihrer Persönlichkeit.

Zurzeit werden nur acht einheimische Sprachen im Alphabetisierungsprozess als offizielle Bildungs- bzw. Alphabetisierungssprachen berücksichtigt, nämlich Moore, Fulfuldé, Gulmancéma, Jula, Lyélé, Dagara, Bissa und Nuni. Diese repräsentieren $13 \%$ der burkinischen Nationalsprachen. Es gibt 60 ethnische Gruppen in Burkina Faso und jede Ethnie wird durch ihre Sprache gekennzeichnet. 87\% der Ethnien Burkina Fasos hoffen also, dass ihre Sprachen eines Tages verwendet werden und in ihrer jeweiligen Sprache Lehrwerke oder Zeitungen schreiben zu können. Demnach gibt es immer noch viel zu tun, damit alle burkinischen Sprachen für die Alphabetisierung verwendet werden.

\section{Die Herausforderung der Alphabetisierung in Burkina Faso}

Die Entwicklung der Alphabetisierung in Burkina Faso wird - wie bereits erwähnt - von zwei Phasen bestimmt: Die erste bildet die Periode der 1960er Jahre. Diese entspricht der traditionellen Alphabetisierung, die im Erlernen des Lesens, Schreibens und Rechnens bestand. Die zweite Phase begann mit der Einführung des Versuchs des Weltprogramms der Alphabetisierung auf der in Iran gehaltenen Tagung im Jahre 1965. Diese Phase nennt man funktionale Alphabetisierung. Sie besteht nicht nur im Erwerb von Fähigkeiten wie Lesen, Schreiben und Rechnen, sondern auch daraus das Gelernte in die alltäglichen Tätigkeiten zu integrieren. Solch eine Alphabetisierung dient dazu, die Lebens- und die Arbeitsbedingungen der Ausgebildeten zu verbessern und darüber hinaus einen Beitrag zur Entwicklung des Landes zu leisten. Seit der Erlangung der Unabhängigkeit ist Burkina Faso darum bemüht, Strategien für die Alphabetisierung zu entwickeln. Yaméogo (1995:8) behauptet, dass im Jahre 1960 die Analphabetisierungsquote in Burkina Faso 92\% war. Seitdem sind zahlreiche Initiativen organisatorischer, institutioneller und erzieherischer Art entwickelt worden, um diese Zahl so schnell wie möglich zu reduzieren.

Seit 1961 sind tatsächlich viele Zentren zur ländlichen Erziehung gebaut worden. Kurz nach der Unabhängigkeit hatten diese Zentren zum Ziel, diejenigen Jugendlichen auszubilden, die keine Chance hatten, zur Schule zu gehen. Die Alphabetisierung und die Ausbildung wurden auf Französisch vermittelt und bestand darin, Kenntnisse über die Landwirtschaft zu vermitteln. Diese Zentren wurden zu Ausbildungszentren für junge Landwirte, die 14 bis 16 Jahre alt waren. Im Unterschied zu den anderen Zentren, bekamen diese Jugendlichen eine berufliche Ausbildung in Landwirtschaft und Tierzucht. 
Viele weitere Zentren wie z.B. die Alphabetisierung „Commando“ oder „Bantaare“ wurden gegründet. In der folgenden Tabelle zeigt die Generaldirektion der Alphabetisierung und informellen Erziehung die Entwicklung der Alphabetisierungskampagnen von 2000 bis 2006:

\begin{tabular}{|c|c|c|c|c|c|c|c|}
\hline \multirow[b]{2}{*}{ Jahre } & \multirow[b]{2}{*}{ Geschlecht } & \multicolumn{5}{|c|}{ Ausbildungsniveau } & \multirow[t]{2}{*}{ Total } \\
\hline & & $\mathbf{I A}^{1}$ & $\mathrm{FCB}^{2}$ & FTS $^{3}$ & $\mathbf{A 3 F}^{4}$ & $\mathrm{CST}^{5}$ & \\
\hline \multirow{3}{*}{$\begin{array}{l}2000- \\
2001\end{array}$} & Männer & 29402 & 9103 & & & & 38505 \\
\hline & Frauen & 30776 & 6839 & & & & 37615 \\
\hline & Gesamt & 60178 & 15942 & & & & 76120 \\
\hline \multirow{3}{*}{$\begin{array}{l}2001- \\
2002\end{array}$} & Männer & 45442 & 16886 & & & & 62328 \\
\hline & Frauen & 61198 & 14547 & & & & 75745 \\
\hline & Gesamt & 106640 & 31433 & & & & 138073 \\
\hline \multirow{3}{*}{$\begin{array}{l}2002- \\
2003\end{array}$} & Männer & 69958 & 24409 & 5137 & & & 99504 \\
\hline & Frauen & 84523 & 25120 & 2095 & & & 111738 \\
\hline & Gesamt & 154481 & 49529 & 7232 & & & 211242 \\
\hline \multirow{3}{*}{$\begin{array}{l}2003- \\
2004\end{array}$} & Männer & 83050 & 31701 & 3167 & 874 & 167 & 118959 \\
\hline & Frauen & 99273 & 34228 & 2988 & 512 & 121 & 137122 \\
\hline & Gesamt & 182323 & 65929 & 6155 & 1386 & 288 & 256081 \\
\hline \multirow{3}{*}{$\begin{array}{l}2004- \\
2005\end{array}$} & Männer & 83430 & 43535 & 1974 & 2189 & 187 & 131315 \\
\hline & Frauen & 113982 & 47378 & 1906 & 2421 & 303 & 165990 \\
\hline & Gesamt & 197412 & 90913 & 3880 & 4610 & 490 & 297305 \\
\hline \multirow{3}{*}{$\begin{array}{l}2005- \\
2006\end{array}$} & Männer & 73280 & 52734 & 2412 & 3090 & 354 & 131870 \\
\hline & Frauen & 115195 & 64737 & 3467 & 2344 & 236 & 186079 \\
\hline & Gesamt & 188475 & 117571 & 5879 & 5434 & 590 & 317949 \\
\hline \multirow[t]{3}{*}{ Gesamt } & Männer & 384562 & 178368 & 12690 & 6153 & 708 & 582481 \\
\hline & Frauen & 504947 & 192949 & 10456 & 5277 & 660 & 714289 \\
\hline & Gesamt & 889509 & 371317 & 23146 & 11430 & 1368 & 1296770 \\
\hline
\end{tabular}

Tabelle 1. Statistik der Eingeschriebenen auf verschiedenen Ausbildungsebenen während der letzten sechs Jahre der Alphabetisierungskampagnen.

(Quelle: Rapport général de la campagne d'alphabétisation 2005-2006. P.6, DGAENEF)

Anmerkungen: 1. Anfangsalphabetisierung; 2. Ergänzende Grundausbildung; 3. Spezifische technische Ausbildung; 4. Grundfranzösisch und funktionelles Französisch lernen; 5.Wissenschafts- und Technikkultur.

Im Anschluss daran sind Bildungsprojekte zur elementaren Bildung durch die Verwendung einheimischer Sprachen entstanden, nämlich Satellitenschulen, Gemeinschaftszentren und Zentren für informelle Grunderziehung sowie bilinguale Schulen. Doch eine Alphabetisierung in einheimischen Sprachen reicht nicht aus, denn es ist unentbehrlich, dass die Alphabetisierten auch eine Fremdsprache wie Französisch lernen. In diesem Fall könnten sich die in einheimischen Sprachen alphabetisierten Jugendlichen für Übersetzungstätigkeiten interessieren. Da die wichtigsten Informationen über Landwirtschaftstechniken auf Französisch verfasst sind, könnten die Alphabetisierten Zugang zu „Originalinformationen“ haben, wenn sie Französisch lesen können. Die Alphabetisierung in einheimischen Sprachen ist zwar erfreulich, aber sehr begrenzt. Die Alphabetisierten beherrschen nur ihre Ethniensprachen, aber haben keine Kenntnisse in den anderen burkinischen Sprachen oder der Fremdsprache Französisch, die die einzige offizielle Sprache des Landes ist. Da die Alphabetisierten aber mit den anderen burkinischen einheimischen Sprachen konfrontiert 
werden, ist es notwendig, dass sie auch die Fremdsprache Französisch lernen um somit die beherrschte Muttersprache zu ergänzen.

Die interessanten Ergebnisse der Generaldirektion der Alphabetisierung und informellen Erziehung könnten verbessert werden, wenn diese einerseits das Erlernen der Fremdsprache Französisch in die Alphabetisierungspläne integrieren und andererseits die informelle Bildung berufsorientiert ausrichten würde, wie im Folgenden dargelegt werden soll.

\section{Berufsorientierung in der informellen Bildung}

Sicherlich sind die Alphabetisierung und die informelle Bildung Schlüssel für die gesellschaftliche Entwicklung. Dennoch ist es sehr schwierig eine zufriedenstellende Alphabetisierungsrate zu erreichen, da es nicht genügend qualifiziertes Lehrpersonal gibt. Da die bilinguale Schule sehr gute Ergebnisse erzeugt, ist dies ein gutes Beispiel dafür, wie die informelle Bildung zu professionalisieren wäre. Diese Berufsorientierung zielt darauf ab, die Qualität der funktionalen Alphabetisierung effizient zu optimieren.

Eine Berufsorientierung in der informellen Bildung ist im heutigen Kontext der Ausdehnung der einheimischen Sprachen in Burkina Faso erforderlich. Die vielfältigen Bemühungen der Regierung haben zur Gründung von Institutionen wie dem Staatssekretariat zur Alphabetisierung und zur informellen Bildung geführt, das später zu einem Stab des parlamentarischen Staatssekretärs wurde. Sie führten auch zur Gründung der Generaldirektion der Alphabetisierung und der informellen Erziehung. Ein Fonds zur Unterstützung der informellen Bildungsaktivitäten wurde ebenfalls gegründet. Im Hinblick auf die schon existierenden Bestimmungen ist es an der Zeit, eine Abteilung für die einheimischen Sprachen an der Universität Koudougou zu eröffnen. Genauso wäre es in den formalen Schulen interessant, von diesem Modell auszugehen, um Lehrerausbildungsvorschläge zu machen und darüber hinaus eine Spezialinspektion für die Alphabetisierung und die informelle Bildung zu gründen.

In der Tat gibt es in der Pädagogischen Hochschule der Universität Koudougou schon eine Abteilung für die Grundschulausbilder (Lehrer, Fachberater, Inspektoren). Der vorläufige Lehrplan für jede Ausbildungsstufe enthält ein bilinguales Lehren und Lernen. Von insgesamt 1.585 Ausbildungsstunden sind den drei Modulen der bilingualen Erziehung, der Alphabetisierung und der informellen Bildung 130 Stunden gewidmet. Diese Stundenzahl weist darauf hin, dass die einheimischen Sprachen eine wichtige Rolle bei der Ausbildung der Grundschulbetreuer spielen.

Die Ausbildung besteht aus vier Modulen:

$\checkmark$ Curricula der Zentren zur bilingualen und informellen Bildung (25 Stunden)

$\checkmark$ Nationalsprachen, formale und informelle Bildung in Burkina Faso (25 Stunden)

$\checkmark$ Transkription der Nationalsprachen (40 Stunden)

$\checkmark$ Didaktik der Nationalsprachen (40 Stunden)

Es ist wichtig hinzuzufügen, dass die Auszubildenden darüber klagen zu wenig Unterricht zu bekommen, weil diese Summe an Unterrichtsstunden unzureichend ist, die einheimischen Sprachen und ihre Didaktik zu erlernen. 
Da es viele spezifische Zentren für die informelle Bildung und die Alphabetisierung gibt, wäre es sinnvoll, spezifische Lokalsprachenlehrer und Lokalsprachenbetreuer in der Pädagogischen Hochschule für die oben genannten Zentren und die bilingualen Schulen vorzubereiten. Folgende Ausbildungsmöglichkeiten wären denkbar:

$\checkmark$ Assistenten zur informellen Erziehung

$\checkmark$ Fachberater zur informellen Erziehung

$\checkmark$ Inspektoren zur informellen Erziehung

Im formalen Erziehungssystem entsprechen die vorgeschlagenen spezifischen Berufe der informellen Erziehung jeweils den folgenden Ausbildungsstufen:

$\checkmark$ Grundschullehrer der klassischen Schule

$\checkmark$ Grundschulfachberater der klassischen Schule

$\checkmark$ Grundschulinspektor der klassischen Schule

Was die Ausbildungsdauer betrifft, sind neun Monate für die theoretische Ausbildung in der Pädagogischen Hochschule der Universität Koudougou und ein anderes Schuljahr für das Praktikum vorgesehen.

Die Inspektoren der informellen Bildung können eine Abschlussarbeit am Ende der Ausbildung schreiben, genauso wie in der klassischen Ausbildung. Interessanter wäre es jedoch, diese Abschlussarbeit in den jeweiligen Nationalsprachen zu schreiben.

\section{Schlussfolgerung}

Zusammenfassend lässt sich festhalten, dass die Analphabetenrate in Burkina Faso immer noch zu hoch ist, was einer der Hauptgründe der Unterentwicklung und der Armut ist. Dennoch bemüht sich die burkinische Regierung durch das Grundschulministerium darum, die Analphabetenrate stark zu reduzieren. Da die Analphabetenrate zu hoch ist, ist das Budget für die informelle Bildung und die Alphabetisierung zweifelsohne ungenügend. Dank der Unterstützung der Nicht-Regierungsorganisation des Schweizerischen Arbeiterhilfswerks und weiteren Partnern werden viele Alphabetisierungszentren und Zentren zur informellen Bildung gegründet. Die TeilnehmerInnen dieser Ausbildung lernen nicht nur ihre eigenen Sprachen kennen, sondern auch die Fremdsprache Französisch. Nach den Statistiken der eingeschriebenen Lernenden auf den verschiedenen Ausbildungsebenen während der letzten sechs Jahre der Alphabetisierungskampagnen, sowie der Situation der bilingualen Schulen von 1994 bis 2007 können wir hoffen, dass sich die Analphabetenrate allmählich reduziert, weil die Analphabeten an der formalen Alphabetisierung interessiert sind.

Von diesen interessanten Ergebnissen ausgehend, wäre eine spezifische Lehrer-, Fachberaterund Inspektorenausbildung an der Pädagogischen Hochschule der Universität Koudougou sinnvoll, um die Lernenden in den informellen Schulen besser auszubilden. Diese anvisierte Professionalisierung der informellen Erziehung würde darauf abzielen, dem Beruf der Nationalsprachenlehrer, -fachberater und -inspektoren einen besonderen Stellenwert in dem burkinischen Schulsystem zu geben. Dadurch könnten die Nationalsprachen in der burkinischen Verwaltung mündlich und schriftlich eine größere Bedeutung erlangen und darüber hinaus könnten die informelle Bildung und die funktionale Alphabetisierung zur Entwicklung des Landes beitragen. Sonst geht verloren, was die Schüler in den informellen Schulen gelernt haben. 
Das o.g. Ausbildungsprojekt kann sich erst effizient verwirklichen, wenn die einheimischen Sprachen in die Curricula der Sekundarschulen integriert werden. Von der Grundschule bis zur Universität sollten Fremdsprachen und Nationalsprachen miteinander verzahnt unterrichtet werden. Dies würde nicht nur zur Beherrschung der Fremdsprache und der einheimischen Sprachen, sondern auch einem Verständnis der fremden und der eigenen Kultur beitragen.

\section{Bibliographie}

Bationo, J-C. 2006. Der burkinische Deutschunterricht auf der Suche nach Identität. Mont Cameroun N3: 49-61.

Besse, J-M. 2003. Qui est illettré? Décrire et évaluer les difficultés à se servir de l'écrit. Paris: Editions Retz.

DGAENF. 2006. Projet de politique générale éditoriale en AENF. Ouagadougou. Maschinenschrift.

DGAENF. 2006. Rapport Général de la campagne 2005-2006. Ouagadougou. Maschinenschrift.

DGINA. 1999. Etude des actions convergentes pour atteindre un taux d'alphabétisation de $40 \%$ d'ici l'an 2009. Ouagadougou. Maschinenschrift.

Geffroy, M-T. et V. Grasset-Morel. 2003. L'illetrisme. Mieux comprendre pour agir. Toulouse: Editions Milon.

Ilboudo, P.T. 2006. L'éducation bilingue au Burkina Faso. Ouagadougou. Maschinenschrift.

Kinda, L. 2009. L'alphabétisation en milieu de travail selon l'approche par les compétences: Etat des lieux et perspectives. Mémoire de fin de formation IEPD. Université de Koudougou. Maschinenschrift.

Ki-Zerbo, J. 1990. Eduquer ou périr. Paris: Unicef-Unesco.

Lepeytre, J. et E. Parra-Ponce. 2008. Lutter ensemble contre l'illettrisme. Paris: Editions Autrement.

UNESCO 2007. L'éducation pour tous en 2005. Un objectif accessible? Paris: Editions UNESCO.

UNESCO. 2007. Taux d'alphabétisation en Afrique. Online verfügbar unter http://www.statistiquesmondiales.com/ alphabetisation.htm.

Yaméogo, A. 1995. L’alphabétisation au Burkina Faso. Ouagadougou. Maschinenschrift.

Zono, A. 1998. Muttersprachlicher Grundschulunterricht in Burkina Faso. Eine soziolinguistische Untersuchung von Modellversuchen. Aachen: Shaker Verlag.

\section{Biographical note}

Born in 1971. Lecturer in studies of language didactics and pedagogics at the Pedagogic College Koudougou (Burkina Faso). Teacher of German at the gymnasium; DEA at the University of Metz (France); received his Ph.D. at the Universität des Saarlandes; maitre assistant at the University Koudougou and the University Ougadougou. Main research: foreign languages and literature didactics, intercultural studies, comparative analyses of present German and African literature, literary anthology, literary translation didactics. 